\title{
Strong coupling between localized and propagating plasmon polaritons
}

\author{
Sinan Balcl, ${ }^{1, *}$ Ertugrul Karademir, ${ }^{2}$ and Coskun Kocabas ${ }^{2}$ \\ ${ }^{1}$ Department of Astronautical Engineering, University of Turkish Aeronautical Association, 06790 Ankara, Turkey \\ ${ }^{2}$ Department of Physics, Bilkent University, 06800 Ankara, Turkey \\ *Corresponding author: sbalci@thk.edu.tr
}

Received 12 May 2015; revised 11 June 2015; accepted 11 June 2015; posted 12 June 2015 (Doc. ID 240820); published 30 June 2015

We investigate plasmon-plasmon (PP) coupling in the strongly interacting regimes by using a tunable plasmonic platform consisting of triangular $\mathrm{Ag}$ nanoprisms placed nanometers away from $\mathrm{Ag}$ thin films. The nanoprisms are colloidally synthesized using a seed-mediated growth method and having size-tunable localized surface plasmon polariton (SPP) resonances immobilized on $\mathrm{Si}_{3} \mathbf{N}_{4}$ films. The PP coupling between the localized SPPs of metal nanoprisms and the propagating SPPs of the metal film is controlled by the nanoprism concentration and the plasmon damping in the metal film. Results reveal that Rabi splitting energy determining the strength of the coupling can reach up to several hundreds $\mathrm{meV}$, thus demonstrating the ultrastrong coupling occurring between localized and propagating SPPs. The metal nanoparticle-metal thin film hybrid system over the square-centimeter areas presented here provides a unique configuration to study PP coupling all the way from the weak to ultrastrong coupling regimes in a broad range of wavelengths. (๑) 2015 Optical Society of America

OCIS codes: (240.0240) Optics at surfaces; (240.5420) Polaritons; (240.6680) Surface plasmons; (250.5403) Plasmonics; (260.0260) Physical optics.

http://dx.doi.org/10.1364/OL.40.003177

Studying light-matter interaction at the nanoscale dimension with metallic nanostructures and metallic thin films has become an intense area of research due to the ability of metallic nanostructures and metallic ultrathin films to confine and concentrate light at the subwavelength scale by excitation of surface plasmon polaritons (SPPs) [1,2]. SPP-enabled enhancement of light-matter interaction has been further driven by the developments of improved top-down and bottom-up nanofabrication techniques and also by the ability to chemically synthesize quantum dots with size- and shape-tunable optical properties.

To further widen the scope of the fundamental and applied science application of metallic plasmonic structures and boost the performance of the plasmonic devices, new hybrid platforms with easily tunable optical properties are required for engineering light-matter interaction at the nanoscale. This goal has been sought either by metal nanoparticle-metal film [3-11] or metal nanoparticle-metal nanoparticle [12-15] configurations. Previously, metal nanoparticles placed nanometers away from continuous metal thin films resulting in a number of enhanced optical effects and tunable plasmon resonances have been proposed and experimentally demonstrated as plasmonic platforms to study coupling of localized SPPs (LSPP) and propagating SPPs (PSPPs) [3-11]. Until now, the plasmonplasmon (PP) coupling observed in this configuration has been mostly studied using isotropic Ag nanoparticles having plasmon resonance at $\sim 400 \mathrm{~nm}$ or isotropic gold nanoparticles having plasmon resonance at $\sim 530 \mathrm{~nm}$. In other cases, metal nanodisks and gratings have been used to study the PP coupling $[9,10]$. An optimum dielectric spacer layer thickness of around $10-30 \mathrm{~nm}$ was found to maximize PP coupling efficiency [3]. In the previous studies: (1) PP coupling has been investigated in the strong coupling regime, nevertheless, the extent of the coupling has not been reported; (2) most of coupling observations have been performed by only exciting the LSPP of the nanoparticles; thus, it is interesting to study the coupling by exciting the PSPPs of the metal film; (3) most of the hybrid samples have been fabricated by using lithographic techniques; thus, finding a complementary plasmonic platform with easily tunable plasmonic properties is appealing for a variety of surfaceenhanced optics applications.

Herein, we report the PP coupling in a tunable plasmonic platform formed by chemically synthesized Ag triangular nanoprisms (Ag NPs) with size-tunable optical properties placed a few nanometers away from Ag thin films, as depicted in the schematic representation [Figs. 1(a) and 1(b)]. The hybrid system presented here covers a square-centimeter area and provides a unique configuration to study PP coupling all the way from the weak to ultrastrong coupling regimes in a broad range of wavelengths. Different from the previous studies, we seek to improve on the optical properties of the hybrid system and find that the hybrid system studied here has the following properties: (1) it has tunable plasmonic properties; (2) plasmon damping of Ag films and concentration of Ag NPs can be used 

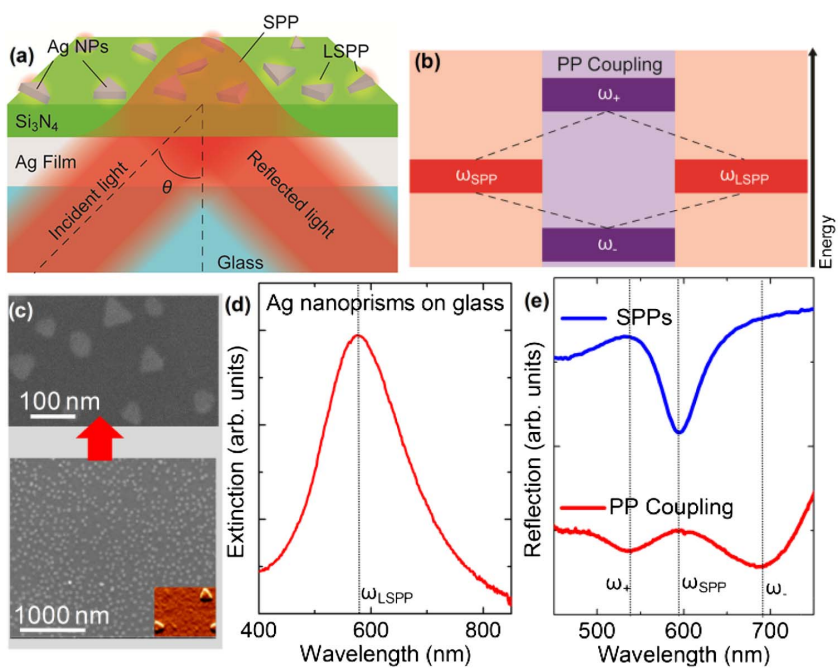

Fig. 1. (a) Schematic representation of the experimental setup used to measure reflections in the Kretschmann configuration. Reflection measurements are made over $40^{\circ}-50^{\circ}$ with a $\sim 0.2^{\circ}$ angle increment. (b) Schematic representation of the PP coupling. (c) SEM images of uniformly coated Ag NPs on APTES modified silicon substrate. The inset indicates an AFM image of the Ag NPs. (d) Extinction spectrum of Ag NPs. (e) Reflection spectra obtained from a bare Ag thin film and Ag NPs coated Ag film at around $45^{\circ}$ incidence angle. The upper and lower polariton bands are at $\sim 525$ and $\sim 650 \mathrm{~nm}$, respectively.

for tuning optical properties of the hybrid system; (3) the system exhibits very large Rabi splitting energies for thick Ag films, which is first due to the decreased plasmon damping observed in the large Ag film thicknesses and, second, due to the ability of Ag NPs to localize high electric fields at their sharp corners [16-20].

Triangular-shaped Ag NPs $(0.02 \mathrm{mg} / \mathrm{ml})$ are rapidly synthesized via seed mediated method at room temperature, and details of the synthesis can be found in our previous works $[\underline{16}, \underline{17}]$. A series of $\mathrm{Ag}$ thin films with different thicknesses are prepared onto glass substrates using thermal evaporation technique. Dielectric spacer layer of $\mathrm{Si}_{3} \mathrm{~N}_{4}$ is deposited by $\mathrm{RF}$ magnetron sputtering in $\mathrm{Ar}$ atmosphere. Prior to immobilization of Ag NPs, the surfaces are modified with 3-aminopropyltriethoxysilane (APTES) (10 $\mathrm{mM}$ in ethanol) [16]. Subsequently, the Ag NPs were allowed to immobilize on the functionalized surfaces for $24 \mathrm{~h}$ [Fig. 1(c)]. To understand the optical properties of the bare Ag NPs, they are placed on glass surfaces. The spectrum reveals that Ag NPs have LSPPs at around $600 \mathrm{~nm}$ [Fig. 1(d)]. Previous theoretical studies showed that the Ag NPs' extinction spectrum strongly depends on the edge length and thickness [19]. Recent experimental results have also confirmed the theoretical calculations and the position of the main plasmon resonance can be expressed as $\lambda_{\max }=33.8$ (edge length/thickness) +418.8 [20]. Since we measured the average thickness of the Ag NPs as $\sim 5 \mathrm{~nm}$ by using atomic force microscopy (AFM), the main plasmon resonance position $(\sim 600 \mathrm{~nm})$ and edge length of the Ag NPs $(\sim 27 \mathrm{~nm})$ can be easily correlated [Fig. 1(d)]. The reflection measurements in transverse-magnetic $(\overline{\mathrm{TM}})$ mode are performed using an ellipsometer in the Kretschmann configuration $[\underline{18}, 21]$. The prism couples the incident light that is $\sim 2 \mathrm{~mm}$ in diameter to free electrons oscillating on the metal surface. This is observed when the horizontal component of the TM polarized light $\left(k_{x}\right)$ is equal to the real part momentum of SPPs $\left(k_{\text {spp }}\right)$ :

$$
k_{x}=k_{0} n_{p} \sin (\theta)=k_{\text {spp }}=\frac{2 \pi}{\lambda} \sqrt{\frac{\varepsilon_{m} \varepsilon_{d}}{\varepsilon_{m}+\varepsilon_{d}}},
$$

where $\lambda$ is the wavelength of the incident light, $k_{0}$ is the wave vector of the incident light, $n_{p}$ is the refractive index of the prism used to excite SPPs, $\theta$ is the plasmon resonance angle, $\varepsilon_{d}$ and $\varepsilon_{m}$ are the dielectric constants of dielectric and metal, respectively. Polarization dependent reflection spectra from a bare $\mathrm{Ag}$ film and $\mathrm{Ag}$ NPs coated $\mathrm{Ag}$ film reveal that two new optical modes at $\sim 525$ and $\sim 650 \mathrm{~nm}$ are generated [Fig. 1(e)].

The plasmonic properties of a bare metal film with a dielectric spacer layer have been investigated [Fig. 2(a)]. The dispersion curve of the $40 \mathrm{~nm}$ thick Ag film reveals that PSPP resonance can be tuned as expected by varying only the angle of incident light, Fig. 2(a). For convenience, the plots of the experimentally obtained dispersion curves are expressed as wavelength versus incidence angle. When the Ag NPs are in close proximity to the Ag film, the coupling of LSPPs and PSPPs is observed, Fig. 2. The anticrossing behavior indicates that the coupling is in the strong coupling regime; hence, two new modes are observed as upper and lower polariton branches. The location of the polariton bands can be adjusted by varying the concentration of the Ag NPs on the surface [Figs. 2(b)-2(e)]. The energy difference between the branches at the zero-detuning called the Rabi splitting energy $[4,5]$ is more than $550 \mathrm{meV}$. The upper and lower polariton modes are localized at the band edges, and they are propagating away from the band edges. Recently, plasmon hybridization theory,
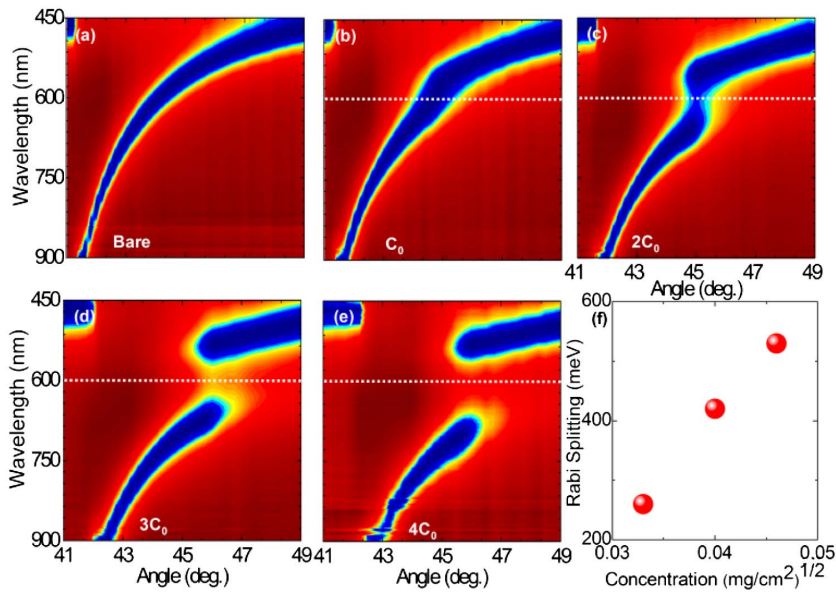

Fig. 2. PP coupling as a function of Ag NPs concentration. (a) SPP reflection curves of $40 \mathrm{~nm}$ thick $\mathrm{Ag}$ film coated with $10 \mathrm{~nm} \mathrm{Si} \mathrm{N}_{3}$. The blue- and red-colored regions indicate the low and high reflectivity, respectively. Polariton reflection curve obtained from the $40 \mathrm{~nm}$ thick Ag film covered with Ag NPs having concentration of (b) $\mathrm{C}_{0}=5.3 \times 10^{-4} \mathrm{mg} / \mathrm{cm}^{2}$; (c) $2 \mathrm{C}_{0}$; (d) $3 \mathrm{C}_{0}$; and (e) $4 \mathrm{C}_{0}$. (f) Rabi splitting energy increases with the square root of Ag NPs concentration as theoretically expected. 
which is an electromagnetic analog of molecular orbital theory, has been developed to understand the plasmon response of complex nanostructures of arbitrary shape [12]. The hybridization of the plasmonic structures generates two new modes as upper polariton branch, $\omega_{+}$, and lower polariton branch, $\omega_{-}$. The $\omega_{+}$mode is at the high-energy side in the energy-level diagram because it is an antisymmetric coupling, whereas the $\omega_{-}$mode is at the lower-energy side in the energy-level diagram since it is a symmetric coupling. Classical description of the strong coupling between PSPPs and Lorentzian oscillator without damping produces two normal modes [2]:

$$
\omega_{ \pm}=\frac{\kappa}{2}+\frac{\omega_{0}}{2} \pm \frac{1}{2} \sqrt{A+\left(\kappa-\omega_{0}\right)^{2}} .
$$

At resonance, when $\kappa=\omega_{0}$, the Rabi splitting becomes

$$
\Omega=\omega_{+}-\omega_{-}=\sqrt{A}=\sqrt{\frac{N}{V}} \frac{e}{\sqrt{\varepsilon_{0} m}},
$$

where $N / V$ is the concentration of the oscillator and $e$ and $m$ are electron charge and mass, respectively. It is clear here that Rabi splitting increases linearly with the square root of the concentration of the oscillator, as we have also seen in Fig. 2(f).

In order to understand the effect of Ag NP size on the PP coupling, Ag NPs with different sizes are immobilized on metal films [Fig. 3]. Polariton reflection curves obtained from the hybrid samples containing Ag NPs having LSPPs at 525, 570, and $600 \mathrm{~nm}$ are shown in Figs. $3(\mathrm{a})-3(\mathrm{c})$, respectively. It is clear that the locations of the lower and upper polariton branches vary with the LSPPs' wavelength [Fig. 3(d)]. Furthermore, by using 3D-FDTD simulations (Lumerical), we have shown that anticrossing behavior (Rabi splitting) can be observed in the same sample configurations used here.

To fully control the strength of the PP coupling, the thickness of the plasmonic layer is varied from 10 to $70 \mathrm{~nm}$ while keeping the thickness of the dielectric spacer layer at $10 \mathrm{~nm}$
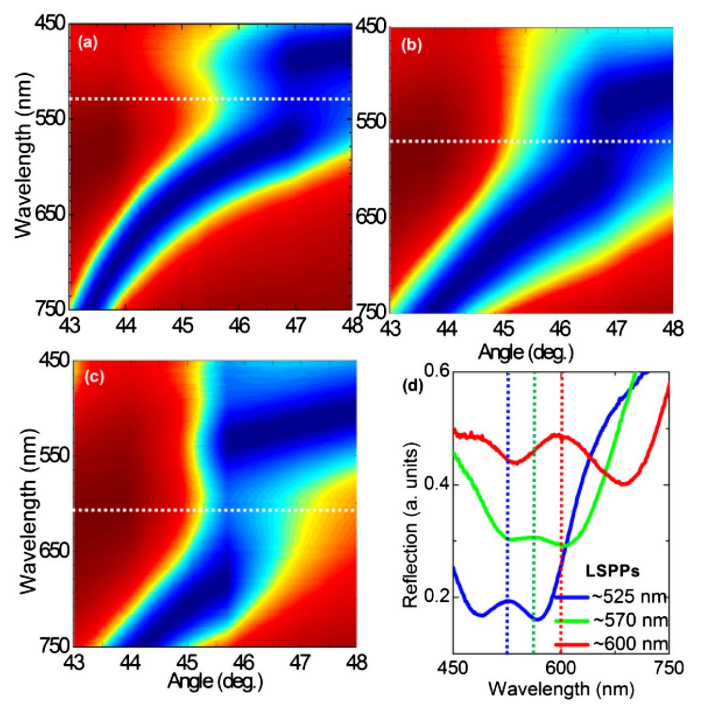

Fig. 3. Polariton reflection curves obtained from the hybrid plasmonic platform having Ag NPs with plasmon resonances at (a) $\sim 525 \mathrm{~nm}$; (b) $\sim 570 \mathrm{~nm}$; and (c) $\sim 600 \mathrm{~nm}$. (d) Locations of the lower and upper polariton branches vary with the LSPPs' wavelength of Ag NPs.
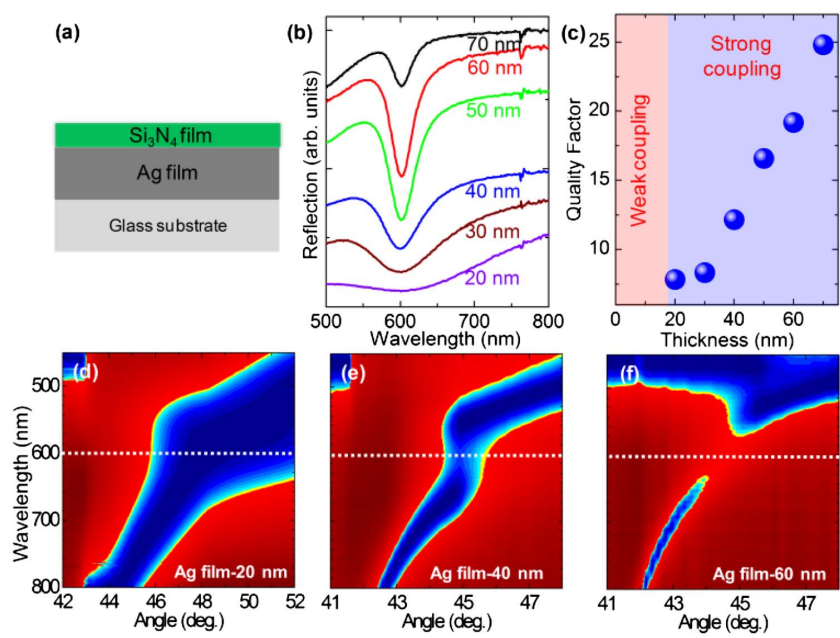

Fig. 4. Tuning quality factor of the PSPP resonance with the Ag film thickness. (a) Schematic representation of the hybrid structure. $10 \mathrm{~nm}$ thick $\mathrm{Si}_{3} \mathrm{~N}_{4}$ film is used as a spacer layer. (b) Reflection spectra of the PSPP resonance at $\sim 600 \mathrm{~nm}$ as a function of film thickness. The incidence angles are varied from $46.8^{\circ}$ to $43.8^{\circ}$ for $\mathrm{Ag}$ film thicknesses ranging from 20 to $70 \mathrm{~nm}$, respectively. (c) The quality factor increases with the metal film thickness. Polariton reflection curves obtained from the hybrid system having $\mathrm{Ag}$ film thicknesses of (d) $20 \mathrm{~nm}$; (e) $40 \mathrm{~nm}$; and (f) $60 \mathrm{~nm}$.

[Fig. 4(a)]. The PSPPs' reflection spectra indicate variation of the resonance peak shape as a function of the plasmonic layer thickness, Fig. $\underline{4(\mathrm{~b})}$. The quality factor $[2,18]$ of the plasmon resonance-describing the plasmon loss relative to the amount of plasmon stored within the metal film-is calculated by dividing the resonance central wavelength by the resonance bandwidth for each plasmonic layer thickness [Fig. 4(c)]. Below $20 \mathrm{~nm}$ Ag film thickness, the damping of the plasmon resonance increases; therefore, the PP coupling enters a completely new regime called the weak coupling regime. For example, at $10 \mathrm{~nm} \mathrm{Ag} \mathrm{film,} \mathrm{the} \mathrm{dispersion} \mathrm{curve} \mathrm{does} \mathrm{not} \mathrm{show} \mathrm{any}$ PP splitting; thus, the coupling is considered to be in the weak coupling regime (no Rabi splitting). Including damping, frequency dependent dielectric constant of metal in Drude model can be expressed as

$$
\varepsilon(\omega)=1-\frac{\omega_{p}^{2}}{\omega^{2}+i \gamma \omega},
$$

where $\gamma$ is the damping rate and $\omega_{p}$ is the plasma frequency of the metal. The damping term is directly proportional to the linewidth of the plasmon resonance and inversely proportional to the quality factor of the plasmon resonance $\{\mathrm{Fig} . \underline{4(\mathrm{c})}$ $[18,21,22]\}$. In the visible spectrum, Ag has a low damping rate, which is why it is preferred in most of the plasmonic applications. In this work, the damping term is tuned by varying the plasmonic layer thickness controlling the observed Rabi splitting. Recent experimental observations have also confirmed our previous results $[18,21]$ in which optical properties of the ultrathin metal films depend on the film thickness [23]. As shown in Fig. 4(b), in order to generate the same plasmon resonance wavelength $(\sim 600 \mathrm{~nm})$ with different $\mathrm{Ag}$ metal film thicknesses, the incidence angle is increased from $46.8^{\circ}$ to 

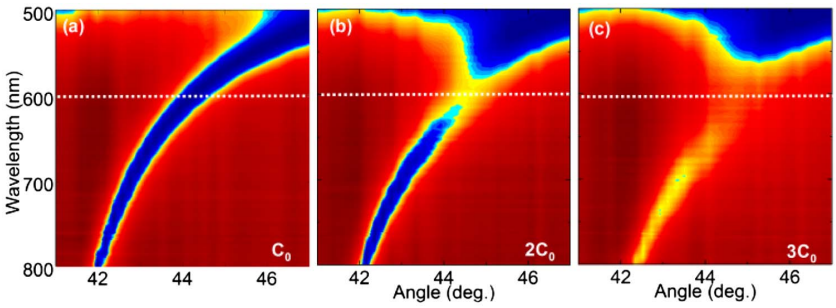

Fig. 5. Ultrastrong PP coupling. Polariton reflection curves obtained from the $60 \mathrm{~nm}$ thick Ag film covered with Ag NPs having concentration of (a) $\mathrm{C}_{0}=5.3 \times 10^{-4} \mathrm{mg} / \mathrm{cm}^{2}$; (b) $2 \mathrm{C}_{0}$; and (c) $3 \mathrm{C}_{0}$. Rabi splitting energy increases with the increase in the $\mathrm{Ag}$ $\mathrm{NP}$ concentration. When the concentration of $\mathrm{Ag} \mathrm{NPs}$ is $3 \mathrm{C}_{0}$, collectively coupled plasmonic modes appear and a large plasmonic bandgap is formed.

$43.8^{\circ}$ for $\mathrm{Ag}$ film thicknesses ranging from 20 to $70 \mathrm{~nm}$, respectively. Concurrently, the plasmonic loss decreases with the Ag film thickness. In the case of dispersion with damping $(\gamma)$, the new normal modes are now [2]

$$
\omega_{ \pm}=\frac{\kappa}{2}+\frac{\omega_{0}}{2}-\frac{i \gamma}{4} \pm \frac{1}{2} \sqrt{A+\left(\kappa-\omega_{0}+\frac{i \gamma}{2}\right)^{2}} .
$$

At resonance when $\kappa=\omega_{0}$, the Rabi splitting becomes

$$
\Omega=\omega_{+}-\omega_{-}=\sqrt{A-\frac{\gamma^{2}}{4}} \text {. }
$$

It is clear in this equation that the Rabi splitting decreases with the increase in the plasmon damping. Indeed, this has been experimentally demonstrated, as seen in Figs. $\underline{4(\mathrm{~d})}-\underline{4(\mathrm{f})}$; Rabi splitting energy increases with the decrease in the plasmon damping in the $\mathrm{Ag}$ film. It should be emphasized here that, in order to observe strong coupling (Rabi splitting) in the dispersion curve, the damping rate of the individual coupling states has to be smaller than the Rabi splitting [2].

To boost the PP coupling observed in Fig. 2 obtained by using $40 \mathrm{~nm}$ thick $\mathrm{Ag}$ film, first, the Ag film thickness is increased to $60 \mathrm{~nm}$; second, the concentration of the $\mathrm{Ag}$ NPs has been increased from $\mathrm{C}_{0}$ to $3 \mathrm{C}_{0}$ where $\mathrm{C}_{0}=5.3 \times$ $10^{-4} \mathrm{mg} / \mathrm{cm}^{2}$ [Figs. 5(a) $-5(\mathrm{c})$ ]. The large energy separation between the lower and upper polariton branches at zero detuning (more than $700 \mathrm{meV}$ ) indicates that the PP coupling is in the strong coupling regime [2]. We believe that increasing the concentration of Ag NPs and quality factor of the Ag thin film results in generation of collectively coupled plasmonic modes [24]; thus, formation of a large plasmonic bandgap can be observed [Fig. 5(c)]. It should be noted here that, when the $\mathrm{Ag}$ film thickness is $40 \mathrm{~nm}$ and covered with $\mathrm{Ag}$ NPs of $3 \mathrm{C}_{0}$ concentration [Fig. 2(e)], Rabi splitting energy of only around $400 \mathrm{meV}$ is observed.

In summary, we have demonstrated that a tunable plasmonic platform consisting of colloidally synthesized Ag NPs placed nanometers distance away from the Ag thin films shows PP coupling ranging from weak to ultrastrong coupling regimes, which are governed by the plasmonic damping and also by the concentration of Ag NPs. In addition, due to the sizetunable plasmon resonance frequency of the Ag NPs, the PP coupling has been investigated as a function of the composition of the hybrid platform. In the strong coupling regime, the hybridization of the plasmonic modes is observed. The PP splitting energy is tunable from a few hundreds $\mathrm{meV}$ to several hundreds $\mathrm{meV}$ (more than $700 \mathrm{meV}$ ) corresponding to more than $35 \%$ of the bare plasmon resonance energy. The tunable plasmonic platform concurrently bearing the optical properties of LSPPs and PSPPs can enhance a variety of optical processes at nanoscale dimension and is, therefore, promising for a wide variety of optical applications at nanoscale dimension. Especially, we believe that the appearance of collectively coupled plasmonic modes appearing at large Ag NPs concentration and large Ag film thicknesses [Figs. 5(a)-5(c)] is interesting for solar cell and surface-enhanced optics applications. The similar investigations also can be performed by exciting the PSPPs using metal grating nanostructures.

Funding. Scientific and Technological Research Council of Turkey (TUBITAK) (112T091).

Acknowledgment. We thank Osman Balci for his help in SEM measurements.

\section{REFERENCES}

1. W. L. Barnes, A. Dereux, and T. W. Ebbesen, Nature 424, 824 (2003).

2. P. Torma and W. L. Barnes, Rep. Prog. Phys. 78, 013901 (2015).

3. W. R. Holland and D. G. Hall, Phys. Rev. B 27, 7765 (1983).

4. J. J. Mock, R. T. Hill, A. Degiron, S. Zauscher, A. Chilkoti, and D. R. Smith, Nano Lett. 8, 2245 (2008).

5. M. Hu, A. Ghoshal, M. Marquez, and P. G. Kik, J. Phys. Chem. C 114, 7509 (2010)

6. A. Christ, T. Zentgraf, S. G. Tikhodeev, N. A. Gippius, J. Kuhl, and H. Giessen, Phys. Rev. B 74, 155435 (2006).

7. A. Farhang, N. Bigler, and O. J. F. Martin, Opt. Lett. 38, 4758 (2013).

8. B. Gallinet, T. Siegfried, H. Sigg, P. Nordlander, and O. J. F. Martin, Nano Lett. 13, 497 (2013).

9. W. Zhou, J. Y. Suh, Y. Hua, and T. W. Odom, J. Phys. Chem. C 117, 2541 (2013).

10. Y. Chu, M. G. Banaee, and K. B. Crozier, ACS Nano 4, 2804 (2010).

11. Y. Chu and K. B. Crozier, Opt. Lett. 34, 244 (2009).

12. E. Prodan, C. Radloff, N. J. Halas, and P. Nordlander, Science $\mathbf{3 0 2}$, 419 (2003).

13. M. Hentschel, M. Saliba, R. Vogelgesang, H. Giessen, A. P. Alivisatos, and N. Liu, Nano Lett. 10, 2721 (2010).

14. M. Ringler, A. Schwemer, M. Wunderlich, A. Nichtl, K. Kürzinger, T. A. Klar, and J. Feldmann, Phys. Rev. Lett. 100, 203002 (2008).

15. J. R. Krenn, A. Dereux, J. C. Weeber, E. Bourillot, Y. Lacroute, J. P. Goudonnet, G. Schider, W. Gotschy, A. Leitner, F. R. Aussenegg, and C. Girard, Phys. Rev. Lett. 82, 2590 (1999).

16. S. Balci, Opt. Lett. 38, 4498 (2013).

17. S. Balci, C. Kocabas, B. Kucukoz, A. Karatay, E. Akhuseyin, H. G. Yaglioglu, and A. Elmali, Appl. Phys. Lett. 105, 051105 (2014).

18. S. Balci, C. Kocabas, S. Ates, E. Karademir, O. Salihoglu, and A. Aydinli, Phys. Rev. B 86, 235402 (2012).

19. K. L. Shuford, M. A. Ratner, and G. C. Schatz, J. Chem. Phys. 123, 114713 (2005).

20. D. Aherne, D. M. Ledwith, M. Gara, and J. M. Kelly, Adv. Funct. Mater. 18, 2005 (2008)

21. S. Balci, C. Kocabas, E. Karademir, and A. Aydinli, Opt. Lett. 39, 4994 (2014).

22. C. Sonnichsen, T. Franzl, T. Wilk, G. von Plessen, J. Feldmann, O. Wilson, and P. Mulvaney, Phys. Rev. Lett. 88, 077402 (2002).

23. J. Gong, R. Dai, Z. Wang, and Z. Zhang, Sci. Rep. 5, 9279 (2015).

24. C. Hanske, M. Tebbe, C. Kuttner, V. Bieber, V. V. Tsukruk, M. Chanana, T. A. F. König, and A. Fery, Nano Lett. 14, 6863 (2014). 